\title{
Outcome in pediatric acute lymphoblastic leukemia in children and adolescents using contemporary protocols of chemotherapy - experience of a single Pediatric Hematology-Oncology Center
}

Claudia DITA ${ }^{1}$, Anca COLITA ${ }^{2,3}$, Mirela ASAN ${ }^{3}$, Anca GHEORGHE ${ }^{4}$, Cerasela JARDAN ${ }^{2,4}$, Mihaela DRAGOMIR ${ }^{2,5}$, Constantin ARION 2,3

${ }_{1}^{1}$ „Stefan S. Nicolau“ Institute of Virology, Bucharest

${ }_{2}^{2}$,Carol Davila" University of Medicine and Pharmacy, Bucharest

${ }^{3}$ Pediatric Clinic, Fundeni Clinical Institute, Bucharest

${ }^{4}$ Fundeni Clinical Institute, Bucharest

${ }^{5}$ Center of Hematology and Bone Marrow Transplantation, Fundeni Clinical Institute, Bucharest

\section{ABSTRACT}

The authors studied the outcome of a cohort of pediatric acute lymphoblastic leukemia (ALL) patients (33 children and adolescence) diagnosed and treated conforming to modern Chemotherapy Protocols (ALL ICBFM 2002, Interfant 06) in a single Center - Pediatric Clinic, Fundeni Clinical Institute, Bucharest, Romania.

They analyzed the factors which determine the prognosis and the outcome of these patients in the course of multi-agent systemic chemotherapy to stand at the base of these Protocols: initial age, initial leukocyte count, blasts immunophenotype, cytogenetic and molecular abnormalities, initial response to cortisone, risk groups, time to obtain the complet remission, etc. Among the factors they discussed, a great value was proven to have the minimal residual disease (MRD) determination in certain check points of Protocols and revaluation of patients risk conforming to MRD values.

Using the modern Protocols and continuously watching the evolution on therapy enable the authors to obtain results close to those of European and North American Pediatric Hematology Oncology Centers: OS $90.9 \%$ by 40 Mo and EFS $72.7 \%$ at the end of the same period of time.

Keywords: children and adolescents, acute lymphoblastic leukemia, prognosis, minimal residual disease

\section{INTRODUCTION}

It is now widely accepted that pediatric ALL is a biologically heterogeneous disease. (1) This heterogeneity manifests also in what concerns the results of therapy and the outcome.
Modern pediatric ALL treatment Protocols employ risk-based therapy to reduce the toxicity in patients with low risk ALL (i.e. low risk of treatment failure), and to use aggressive therapy for those with a high risk of relapse and therefore an unfavorable outcome. 
Newly diagnosed children are subjected to a Treatment Plan including chemotherapy via systemic and intrathecal administration and supportive care. Radiotherapy is now limited for emergencies like mediastinal mass producing respiratory obstruction, or for high risk patients with central nervous system (CNS) disease. $(2,3)$

The contemporary multidrug chemotherapy regimens are working in three different phases:

Induction (initial 4-6 weeks of therapy), which now achieves remission in over $95 \%$ children with ALL. (1-3) The principles of multiagent CXT are: combination of drugs, low therapeutic index and dose intensity (4). In this period of time, as secondary effects of therapy some patients can develop life-threatening toxicities.

Post-induction therapy - lasts 2-3 years and is used to remove any residual leukemia cells (1). It includes early intensification, re-induction, late intensification and maintenance.

A special attention is given to therapy of sanctuary sites for leukemia cells, primarily CNS involvement. Preventative CNS therapy was a major acquisition in the treatment of childhood ALL. The vast majority of contemporary Protocols have replaced cranial RxT with intrathecal therapy (TIT) and systemic intensification of specific agents (i.e. methotrexate); the risk of relapses at the level of sanctuary organs is now $<5 \%$ in standard-risk (SR) ALL. $(2,5,6)$ Cranial radiation is now reserved to treat patients with CNS disease at the time of diagnosis, and high risk of relapse.

Up to $20 \%$ of affected children treated with modern protocols will however relapse and their prognosis remains unfavorable. (7) A therapeutic breakthrough appears to be hematological stem cell transplantation (HSCT). In case of relapse, HSCT is indicated for high-risk patients (e.g., those who relapse on therapy, or with high MRD after reinduction therapy who have a great risk for systemic relapse). $(1,3)$ HSCT can achieve a 5 -year event-free survival (EFS) in nearly $60 \%$ of all risk patients. (8)

Risk adapted therapy in childhood ALL is relying on risk stratification; we discussed this stratification in a previous paper. (9) Here, we intend to summarize risk factors in pediatric ALL: initial age and leukocyte count, CNS status at diagnosis, immunological subtypes of blast, cytogenetic and molecular abnormalities, response to Prednisone in the first 8 days of therapy induction failure, and - in particular - minimal residual disease (MRD) at the end of induction.

Recent studies shows that an important indicator of prognosis is the response of patient to treatment. It is possible now to quantify MRD using polymerase chain reaction (PCR) (detecting specific gene fusion products or specific arrangements of immunoglobulin/T-cell receptor genes), or flow cytometry, which use the aberrant leukemia cells phenotypes to highlight the presence of residual blast cells at the end of induction, or at other critical points in the course of therapy.

These techniques are 10-100 times more sensitive than morphologic evidence of residual blasts, and can detect as fewas 1 leukemia cell in 10,000 to 100,000 normal cells. $(2,10,11)$ MRD negativity at the end of induction $(<1 / 10,000$ leukemia cells) is considered a marker of good prognosis; MRD positivity at end of induction and persistent or rising MRD levels at later check points in therapy signity an increased risk of relapse. $(12,13)$

\section{ORIGINAL STUDY}

\section{Background}

The aim of this study was to asses the results of therapy in a cohort of new diagnosed pediatric ALL using the contemporary Protocols - ALL IC-BFM 2002 and Interfant 06. (14,15)

We focused on influence of variables like gender, initial leukocyte count, blast's phenotype, associated cytogenetic and molecular anomalies, initial response to cortisone, induction outcome, etc on overall and event-free survival (EFS). Also, we analyzed primary (due to disease) and secondary (due to therapy) complications and their impact on survival. We have to mention that using Protocols, all the patients were subjected to an uniform modality of treatment.

\section{OBJECTIVES}

- Evaluation of impact of general factors as gender, age at diagnosis and period of study;

- Evaluation of effects on outcome of factors related to biological hallmarks of ALL: initial leukocyte count, blast's immunophenotype, cytogenetic and molecular abnormalities);

- Contribution of interim evaluation of patients regarding response to therapy by sensitive check points during therapy: initial response to cortisone (D8), disappearance of blasts in bone marrow (BM) at D15, achievement of complete remission (CR) at the end of induction (D33); 
- Value of detecting minimal residual disease (MRD) at the end of induction and during different phases of therapy for setting the treatment and estimation of prognosis;

- Treatment outcome according to risk groups: standard-risk (SR), intermediate-risk (IR), high-risk (HR);

- The impact of primary and secondary complications on overall-survival (OS) and eventfree survival (EFS).

\section{MATERIALS AND METHODS}

Our study is descriptive and observational (non-interventional). Patients were eligible if their age at inclusion was under 18 year and if they were diagnosed and treated in the Pediatric Clinic, Fundeni Clinical Institute, Bucharest, Romania in the period of time 09.01.2008 12.31.2011.

Exclusion criteria were:

- Presence of physiologic statuses which contraindicate the treatment (pregnancy, breast feeding);

- Refused Protocol/Refused Consent to the Treatment - by family or adolescent patients after they have read the Informed Consent;

- Initiation of cytotoxic therapy in another medical institution and following the treatment for longer than 1 month;

- Treatment in our institution for a shorter period than 30 days;

- Secondary ALL;

- Congenital ALL and other forms with rapid fatal outcome (less than 30 days from diagnosis);

- Lack of complete diagnosis or Protocol completion.

We used the same cohort of patients for assessing the value of newer diagnostic methods for risk stratification and therapy in pediatric ALL.

\section{STUDY OVERSIGHT}

The study was conducted in accordance with the Principles of the Declaration of Helsinki (DoH) (16); it was approved by the local Fundeni Clinical Institute Committee for Bio-Ethics and all families and adolescents $>16$ years old have signed Informed Consent. The study had no sponsors, and the investigators personally collected data and analyzed them. The first author wrote the draft of the manuscript, and all the authors reviewed and made the revisions to submit it for publication.

\section{PATIENTS}

The study analyzed 33 consecutive patients, 17 males (51.51\%) and 16 girls (48.48\%) with ages at diagnosis between 3.5 month and 17.8 years (median age: 4.33 years).

We collected the following data at diagnosis:

- Complete personal and familial history;

- Weight, height, corporal surface and BMI (Body Mass Index);

- Complete physical examination (fever, symptoms and signs of infection, peripheral adenopathies, bleeding, symptoms and signs of CNS involvement, symptoms and signs of other extramedullary infiltration - liver, spleen, testes, cutis, etc).

Laboratory investigations at diagnosis are presented in Table 1. $(2,3)$

TABLE 1. Laboratory and imagistic investigations at diagnosis $(2,3)$

CBC differential

BMP - aspirate, trephine biopsy for morphology,

cytochemistry, immunophenotyping, cytogenetics and molecular biology

ESR, CRP

Liver screening tests = ALT, AST, Bilirubin, GGT

Renal function screening tests: urine, serum creatinine, urea, uric acid

Glucose, triglycerides, cholesterol

Serum and urinary electrolytes

Viral infection markers: EBV, HSV, CMV, HAV, HBV, HCV, HIV

$1 / 2$

Coagulation (PT, AP, INR, aPTT, fibrinogen, FDP, d-dimers)

Blood group

Bacteriology (as clinical indicated): blood cultures, nasal and

pharyngeal, swabs, urine culture, coproculture, skin and

other foci cultures

Thorax Rx (PA, LAT)

Abdominal ultrasound

ECG and echocardiography

LP (cytology)

Abbreviations: $C B C$ : complete blood count; $B M P$ : bone marrow puncture; ESR: erythrocyte sedimentation rate;

CRP: C-reactive protein; ALT: alanine aminotransferase;

AST: aspartate aminotransferase; GGT: gamma-glutamyltranspeptidase; EBV: Epstein-Barr virus; HSV: herpes simplex virus; CMV: cytomegalovirus; HAV: hepatitis A virus; HBV: hepatitis B virus; HCV: hepatitis C virus; HIV: human immunodeficiency virus; PT: prothrombin time; AP: prothrombin activity percentage; INR: International Normalized Ratio; aPTT: activated partial thromboplastin time; FDP: fibrin degradation product;

PA: posteroanterior; LAT: lateral; ECG: electrocardiography; LP: lumbar puncture.

The morphologic diagnosis of ALL was established in presence of $>25 \%$ blasts in bone marrow, using the bone marrow aspirate smears submitted to May-Grünwald-Giemsa (MGG), Periodic acid-Schiff (PAS), $\alpha$-Naphthyl Acetate Esterase and Myeloperoxidase (MPO) colorations. 
Immunophenotyping of blasts was conducted in peripheral blood or bone marrow collections. Peripheral blood and bone marrow aspirate were harvested on EDTA (Ethylene diamine tetraacetic acid), then we proceed to isolation of blasts by density gradient centrifugation using Ficoll-Hypaque. Blasts were subject to flow cytometry using a Becton Dickinson FACS scan (FACS - Fluorescence-activated cell sorting) and the panel of monoclonal antibodies shown in Table 2.

TABLE 2. Panel of monoclonal antibodies for immunophenotyping

Marker of progenitor cells: CD 34

Pan-hematopoietic marker: CD 45

B-cell markers: CD 10, CD 19, CD 20, CD 22, CD 24,C $\mu, s \mu$, s

$\lg \mathrm{M}, \mathrm{k}$ and $\lambda$ hight chains

T/NK cell marker: CD 1a, CD 2, CD 3, CD 4, CD 5, CD 7, CD 8,

CD 16, CD 56, TCR $\alpha \beta / \gamma \delta$

Myelo-monocytic markers: CD 11b, CD 13, CD 14, CD 15, CD

33, CD 64, CD 119

Red cell line marker: Glycophorin A

Platelets marker: CD 61

Abbreviations: $C D$ : cluster of differentiation; NK: natural killer; TCR: T-cell receptor.

Cellular lines were defined by EGIL Score (EGIL 2002 - European Group for the Immunological Characterization of Leukemias). (17) Coexpression of myeloid antigens was defined by simultaneous expression of $\geq 1$ myeloid markers (CD 13, CD 33, CD 65) on more than $20 \%$ of lymphoblasts.

Cytogenetic studies were conducted on chromosomes prepared in 24 hours culture on usual media (RPMI 1640, MEM Eagle, TC-199, IC 65) supplemented with bovine fetal serum (FBS), L-glutamine, antibiotics and phytohemagglutinin (PHA). Karyotypes were realized by standard cytogenetic technique and G-band analysis; chromosomal anomalies were assessed conforming to ISHCN (International System for Human Cytogenetic Nomenclature). We used also conventional fluorescence in situ hybridization (FISH) techniques for identification of specific translocations in blast cells.

Molecular biology studies were done on BM aspirate, after DNA extraction by standard techniques and PCR amplification of DNA fragments of interest, in presence of commercial primers for common ALL translocations/products of gene fusion (BCR-ABL, TEL-AML1, MLL-AF4, SILTAL).
We repeated the complex of analyses previously presented in different check points during therapy: D15, D33, W12, at the beginning and the every phase of therapy and the end of therapy.

\section{Classification}

Our patients were classified in 3 risk groups (SR, IR, HR) conforming to ALL IC-BFM 2002 Protocol Criteria (Table 3 ) and for infants allocation to $S R$ and HR group was made conforming to Interfant 2006 Protocol criteria (Table 4). $(14,15)$

TABLE 3. ALL risk stratification according to IC-BFM 2002 Protocol (13)

\begin{tabular}{|c|c|c|}
\hline $\begin{array}{c}\text { Standard Risk } \\
\text { Group (SR) }\end{array}$ & $\begin{array}{c}\text { Intermediate Risk } \\
\text { Group (IR) }\end{array}$ & $\begin{array}{l}\text { High Risk Group } \\
\text { (HR) }\end{array}$ \\
\hline Age 1-6 yearsand & $\begin{array}{l}\text { Blasts number D8 } \\
<1000 / \mu \mathrm{L}(\mathrm{PGR}) \\
\text { and }\end{array}$ & $\begin{array}{l}\text { Blasts number D8 } \\
>1000 / \mu \mathrm{L}(\mathrm{PPR}) \text { or }\end{array}$ \\
\hline $\begin{array}{l}\text { Leucocyte count at } \\
\text { diagnosis } \\
<20000 / \mu \text { Land }\end{array}$ & $\begin{array}{l}\text { Age }>1 \text { - }>6 \text { years } \\
\text { or }\end{array}$ & $\begin{array}{l}\mathrm{BM}-\mathrm{M} 2, \mathrm{M} 3 \\
\mathrm{D} 33 \text { or }\end{array}$ \\
\hline $\begin{array}{l}\text { Blasts number D8 } \\
<1000 / \mu \mathrm{L}(\mathrm{PGR}) \\
\text { and }\end{array}$ & $\begin{array}{l}\text { Leucocyte count at } \\
\text { diagnose } \\
>20000 / \mu \text { Land }\end{array}$ & $\begin{array}{l}\text { Presence of } \mathrm{t}(4 ; 11) \\
\text { / MLL-AF4 or }\end{array}$ \\
\hline $\begin{array}{l}\mathrm{BM}-\mathrm{M} 1, \mathrm{M} 2, \mathrm{D} 15 \\
\text { and }\end{array}$ & $\begin{array}{l}\mathrm{BM}-\mathrm{M} 1, \mathrm{M} 2, \mathrm{D} 15 \\
\text { and }\end{array}$ & $\begin{array}{l}\text { Presence of } \mathrm{t}(9 ; 22) \\
\text { / BCR-ABL or }\end{array}$ \\
\hline \multirow[t]{4}{*}{$\begin{array}{l}\text { BM - M1, D33 } \\
\text { (complete } \\
\text { remission - CR) }\end{array}$} & $\mathrm{BM}$ - M1, D33 or & $\begin{array}{l}\text { Patient in the } \\
\text { IR group and } \\
\text { BM }-\mathrm{M} 3, \mathrm{D} 15 \\
\text { (induction failure) }\end{array}$ \\
\hline & SR criteria & \\
\hline & but BM - M3, D15 & \\
\hline & and $\mathrm{BM}-\mathrm{M} 1, \mathrm{D} 33$ & \\
\hline
\end{tabular}

Abbreviations: PGR: Prednisone good responders; PPR: Prednisone poor responders; $B M-M 1 / 2 / 3$ : BM status according to morphology, M1 (<5\% blasts), M2 (5-24\% blasts) and M3 (greater than or equal to $25 \%$ blasts); BM: bone marrow; D8,15,33: day 8,15,33.

TABLE 4. Risk group definition recording to Interfant 2006 Protocol (14)

\begin{tabular}{|l|l|l|}
\hline $\begin{array}{l}\text { Standard Risk } \\
\text { (SR) }\end{array}$ & \multicolumn{1}{|c|}{ Medium Risk (MR) } & High Risk (HR) \\
\hline $\begin{array}{l}\text { No MLL } \\
\text { rearrangement }\end{array}$ & MLL status unknown or & $\begin{array}{l}\text { MLL } \\
\text { rearrangement } \\
\text { and }\end{array}$ \\
\hline & $\begin{array}{l}\text { MLL rearrangement and } \\
\text { age }>6 \text { months (183 } \\
\text { days) or }\end{array}$ & $\begin{array}{l}\text { Age }<6 \text { months } \\
\text { (183 days) and }\end{array}$ \\
\hline $\begin{array}{l}\text { MLL rearrangement and } \\
\text { age }>6 \text { months (183 } \\
\text { days) andLeukocyte } \\
\text { count at diagnosis } \\
<300,000 / \mu \mathrm{L}\end{array}$ & $\begin{array}{l}\text { Leukocyte count } \\
\text { at diagnosis } \\
\geq 300,000 / \mu \mathrm{L}\end{array}$ \\
\hline
\end{tabular}

Abbreviations: MLL: Myeloid/lymphoid or mixed-lineage leukemia gene.

\section{Treatment}

Multiagent systemic chemotherapy and intrathecal therapy (TIT) was deployed conforming to Protocols above mentioned and depicted in Figure 1 and Figure 2. $(14,15)$ 


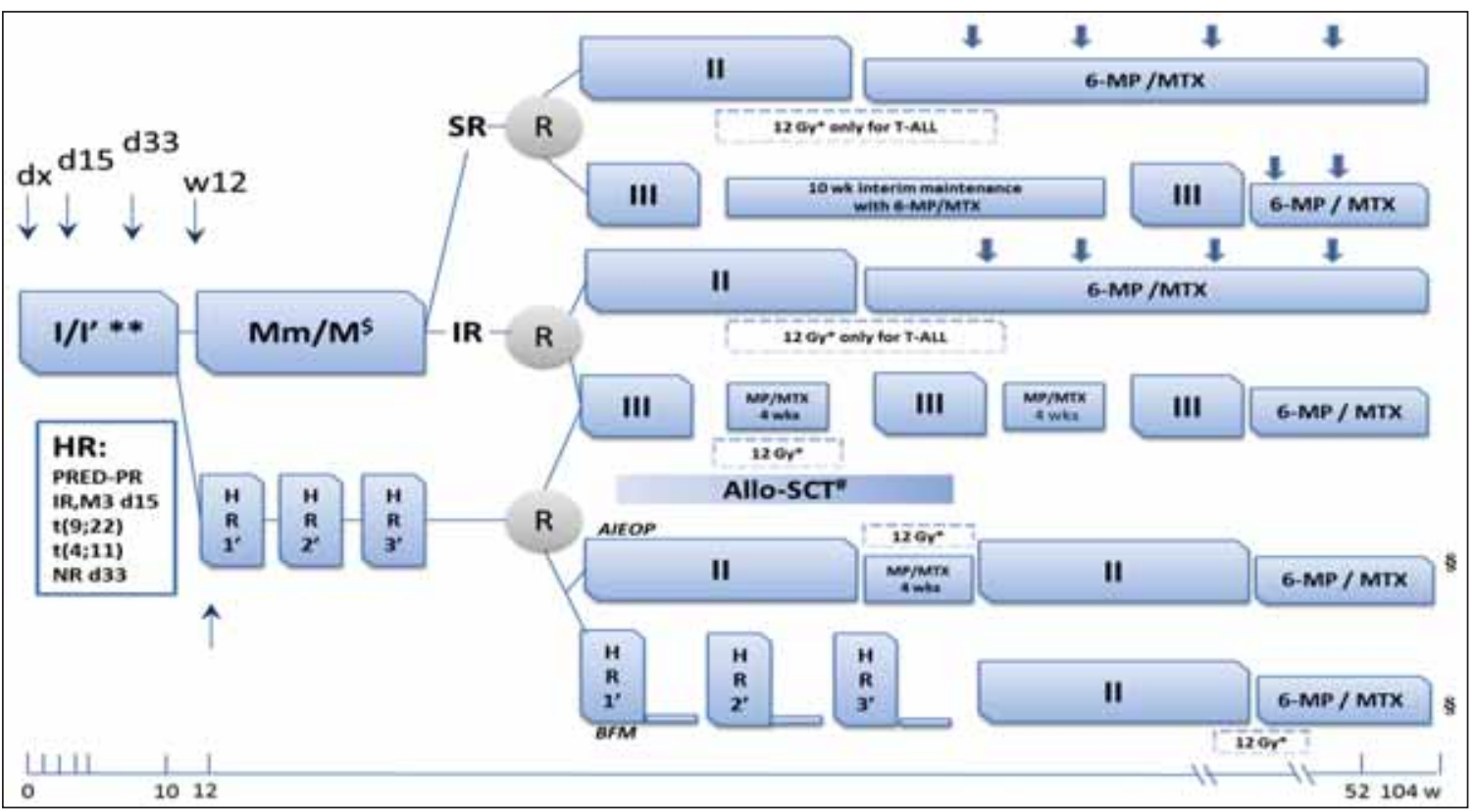

FIGURE 1. Outline of ALL IC-BFM 2002 (Acute Lymphoblastic Leukemia Intercontinental Berlin-Frankfurt-Münster [BFM] 2002) protocol [13].

$\downarrow \downarrow$ BM sampling; $\downarrow$ IT MTX (in maintenance therapy); Protocol I: standard-risk (SR) T-cell acute lymphoblastic leukemia (T-ALL), all intermediate-risk (IR) and high-risk (HR) patients; protocol I': SR B-cell precursor (BCP) -ALL only; protocol M: only T-ALL, SR/IR; protocol mM: only BCP-ALL, SR/IR. * presymptomatic cranial irradiation; **Protocol 1', daunorubicin(DNR) 30mg/ $/ \mathrm{m}^{2} \times 2$ only for SR patients with BCP-ALL; \# selected indications for allogeneic stem-cell transplantation (allo-SCT) in all strata of HR; $\$$ for BCP-ALL,: methotrexate (MTX) $2 \mathrm{~g} / \mathrm{m}^{2} / 24 \mathrm{~h} \times 4$, for T-ALL: MTX $5 \mathrm{~g} / \mathrm{m}^{2} / 24 \mathrm{~h} \times 4$; ${ }^{5}$ No randomization of Associazione Italiana Ematologia Oncologia Pediatrica (AIEOP) vs BFM but choice by group according to previous experience with one of the two HR strategies in trial 95; HR-1', consolidation block HR-1'; HR-2', consolidation block HR-2'; HR-3', consolidation block HR-3'; II, III, protocol designations; dx, diagnosis; d, day; w, week; IT, intrathecal; NR, nonresponder; PRED-PR, prednisone poor response; $R$, randomization;6-MP, mercaptopurine; $M T X$, methotrexate; BM, bone marrow.

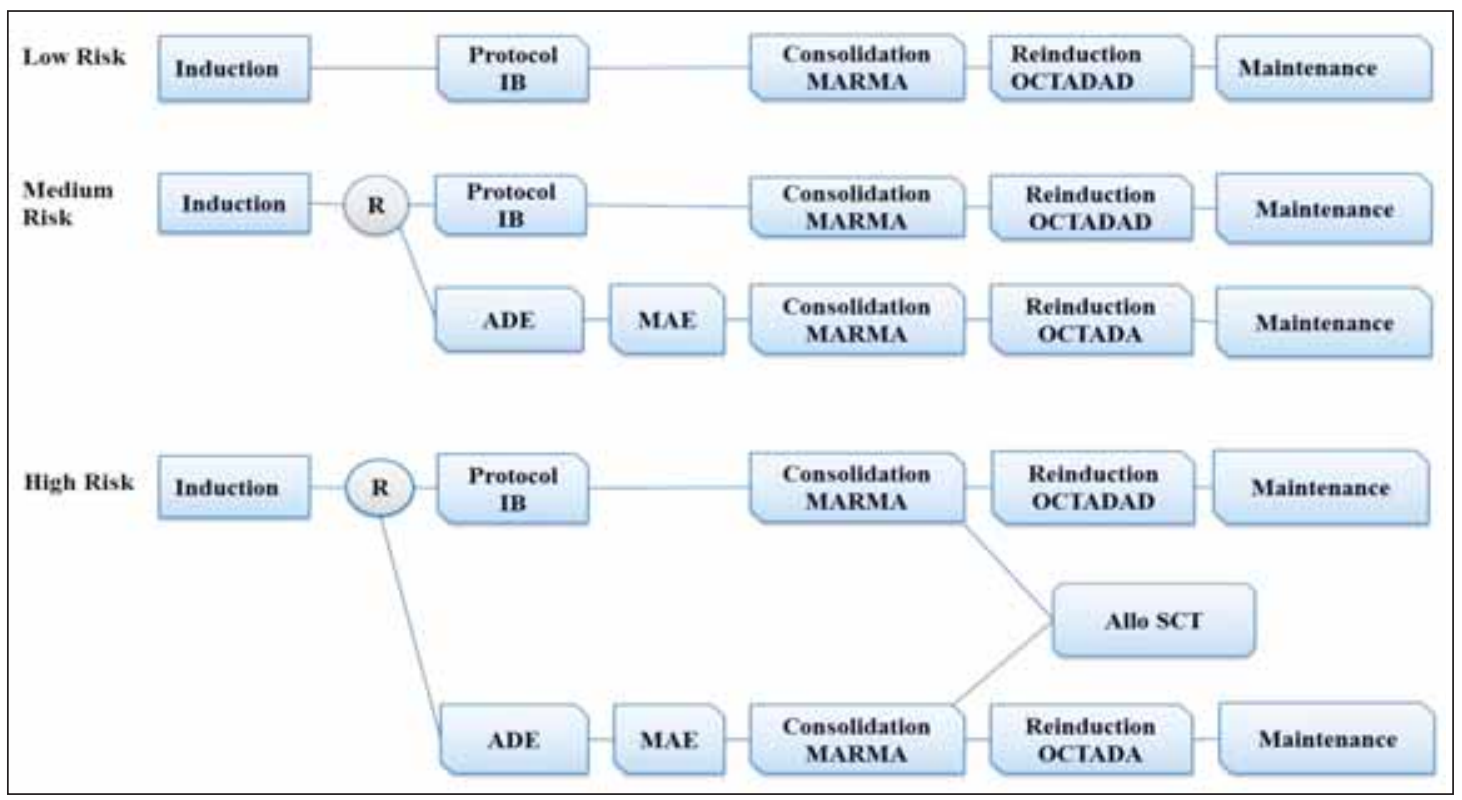

FIGURE 2. Outline of INTERFANT-06 protocol [14]

Induction, PRED PO/IV $60 \mathrm{mg} / \mathrm{m}^{2} /$ day DEXA PO/IV $6 \mathrm{mg} / \mathrm{m}^{2} /$ day VCR IV push $1.5 \mathrm{mg} / \mathrm{m}^{2} /$ dose ARA-CIV $30 \mathrm{~min} 75 \mathrm{mg} / \mathrm{m}^{2} /$ dose DNR IV $1 \mathrm{hr} 30 \mathrm{mg} / \mathrm{m}^{2} /$ dose L-ASP IV $1 \mathrm{hr} 10000 \mathrm{U} / \mathrm{m}^{2} /$ dose (E.coli Asparaginase MEDAC) MTX/PRED ITH acc. to age-Only in case of initial CNS involvement. ARA-C/PRED ITH acc. to age BMP; Protocol IB, CPM IV $1 \mathrm{hr} 1000 \mathrm{mg} / \mathrm{m}^{2} /$ dose $6-M P$ PO $60 \mathrm{mg} / \mathrm{m}^{2} /$ day ARA-CIV push $75 \mathrm{mg} / \mathrm{m}^{2} /$ dose MTX/PRED ITH acc. to age ARA-C/PRED ITH acc. to age BMP (only if BMP at day 33 is inconclusive); ADE, ARA-C IV push $100 \mathrm{mg} / \mathrm{m}^{2} /$ dose DNR IV $1 \mathrm{hr} 50 \mathrm{mg} / \mathrm{m}^{2} /$ dose Etoposide IV $4 \mathrm{hrs} 100 \mathrm{mg} / \mathrm{m}^{2} /$ dose ARA-C/ PRED ITH acc. to age BMP; MAE, ARA-CIV push $100 \mathrm{mg} / \mathrm{m}^{2} /$ dose Mitoxantrone IV $1 \mathrm{hr} 12 \mathrm{mg} / \mathrm{m}^{2} /$ dose Etoposide IV $4 \mathrm{hrs} 100 \mathrm{mg} / \mathrm{m}^{2} /$ dose MTX/PRED ITH acc. to age BMP; MARMA, 6-MP PO $25 \mathrm{mg} / \mathrm{m}^{2} /$ day HD-MTX IV 24hrs $5000 \mathrm{mg} / \mathrm{m}^{2} /$ dose Leucovorin-Rescue PO/IV $15 \mathrm{mg} / \mathrm{m}^{2} /$ dose MTX/PRED ITH acc. to age ARA-CIV $3 \mathrm{hrs} 3000 \mathrm{mg} / \mathrm{m}^{2} /$ dose PEG-ASP IV $1 \mathrm{hr} 2500 \mathrm{U} / \mathrm{m}^{2} /$ dose (ONCASPAR) BMP; OCTADA, DEXA PO/IV $6 \mathrm{mg} / \mathrm{m}^{2} /$ day $6-T G$ PO $60 \mathrm{mg} / \mathrm{m}^{2} /$ day VCR IV push $1.5 \mathrm{mg} / \mathrm{m}^{2} /$ dose, PEG-ASP IV $1 \mathrm{hr} 2500 \mathrm{U} / \mathrm{m}^{2} /$ dose (ONCASPAR) ARA-CIV push $75 \mathrm{mg} / \mathrm{m}^{2} /$ dose ARA-C $/$ PRED ITH acc. to age CPM IV $1 \mathrm{hr} 500 \mathrm{mg} / \mathrm{m}^{2} /$ dose BMP; OCTADAD, OCTADA and DNR IV $1 \mathrm{hr} 30 \mathrm{mg} / \mathrm{m}^{2} /$ dose - DNR not for MR/HR patients randomised to the experimental arm; Maintenance, MTX/PRED ITH acc. to age ARA-C/PRED ITH acc. to age 6-MP PO $50 \mathrm{mg} / \mathrm{m}^{2} /$ day MTX PO $20 \mathrm{mg} / \mathrm{m}^{2} /$ week BMP; PRED, prednisone; DEXA, dexamethasone; VCR, vincristine; ARA-C, cytarabine; DNR, daunorubicin; MTX, methotrexate; MTX/PRED, methotrexate and prednisolone;PEG-ASP, PEG-asparaginase; CPM,cyclophosphamide; 6-MP, mercaptopurine; 6-TG, thioguanine; IV, intravenous; PO, per oral; ITH, intrathecal; BMP, bone marrow puncture. 
TABLE 5. Status of ALL patients at the end of study

\begin{tabular}{|c|c|c|c|c|c|c|c|c|}
\hline \multirow{2}{*}{ Risk group } & \multirow{2}{*}{$\begin{array}{c}\text { Number of } \\
\text { patients }\end{array}$} & \multirow{2}{*}{$\begin{array}{c}\text { Percentage } \\
(\%)\end{array}$} & $\begin{array}{c}\text { Number of } \\
\text { patients }\end{array}$ & $\begin{array}{c}\text { Percentage } \\
(\%)\end{array}$ & $\begin{array}{c}\text { Number of } \\
\text { patients }\end{array}$ & $\begin{array}{c}\text { Percentage } \\
(\%)\end{array}$ & $\begin{array}{c}\text { Number of } \\
\text { patients }\end{array}$ & $\begin{array}{c}\text { Percentage } \\
(\%)\end{array}$ \\
\hline SR & 9 & 27.27 & 8 & 88.89 & 1 & 11.11 & 0 & 0 \\
\hline IR & 14 & 42.42 & 12 & 85.71 & 0 & 0 & 2 & 14.28 \\
\hline HR & 10 & 30.30 & 6 & 60 & 2 & 20 & 2 & 20 \\
\hline
\end{tabular}

Abbreviations: SR: standard risk; IR: intermediate risk; HR: high risk; CR: complete remission.

TABLE 6. The leght of OS and EFS in our cohort of patients

\begin{tabular}{|c|c|c|c|c|c|c|c|c|}
\hline \multirow{2}{*}{ Risk group } & \multicolumn{4}{|c|}{ OS (months) } & \multicolumn{4}{c|}{ EFS (months) } \\
\cline { 2 - 10 } & Minimal value & $\begin{array}{c}\text { Maximal } \\
\text { value }\end{array}$ & Average & Median & Minimal value & $\begin{array}{c}\text { Maximal } \\
\text { value }\end{array}$ & Average & Median \\
\hline SR & 1.5 & 32.9 & 18,4 & 20,1 & 1,5 & 32.9 & 18.4 & 20.1 \\
\hline IR & 4.8 & 39.7 & 22.8 & 20.1 & 4.8 & 39.7 & 22.8 & 20.1 \\
\hline HR & 1.7 & 40 & 11 & 14,9 & 1,1 & 40 & 14.1 & 1 \\
\hline
\end{tabular}

Abbreviations: SR: standard risk; IR: intermediate risk; HR: High risk; OS: overall survival; EFS: event-free survival.

\section{Statistical analysis}

Data obtained from individual sheets of patients were subjected to methods of descriptive statistics. Using the IBM SPSS statistics 20, Epi Info and Microsoft Excel 2010, we computed relative frequencies and realized the graphic representations. In order to test statistical signification of differences we used the $\chi 2$, log-rank tests and Cox proportional regression model. (18)

To estimate survival (OS, EFS) we performed survivor analyses and generated the KaplanMeier survival plots. (19)

\section{Personal study - Results}

\section{OS and EFS in our patients}

Table 5 presents the status of ALL patients at the end of study (maximal length of observation -40 months).

Table 6 depicts the length of OS and EFS in our cohort of patients.

\section{Factors influencing the evolution on therapy}

\subsection{General and related to biological features of pediatric ALL}

\section{Gender}

EFS by 40 Mo (months) was $76,5 \%$ in girls, but only $66,8 \%$ in male patients. Due to the small number of patients, we could not asses the statistical significance of this difference.

\section{Age of patients at diagnosis}

The differencesof EFS in various groups of patients depending on the age at diagnosis is presented in Table 7. As it results from this table, the best prognosis was in the 1-6 years of age, while the age group $<1$ years of age had the worst prognosis.
TABLE 7. EFS in different age groups in our patients

\begin{tabular}{|c|c|c|c|}
\hline Age group & EFS* (\%) & $\begin{array}{c}\text { Assessed at } \\
\text { (months) }\end{array}$ & Observations \\
\hline$<1$ yoa & 0 & 8 & The worst prognosis \\
\hline $1-6$ yoa & 83.3 & 40 & The best prognosis \\
\hline $6-12$ yoa & 71.4 & 40 & \\
\hline $12-18$ yoa & 66.7 & 40 & \\
\hline
\end{tabular}

*Number of months from diagnosis to relapse, to death due to the toxicity of therapy or to the abandonment of study.

Abbreviations: yoa: years of age; EFS: event-free survival.

\section{Initial leukocyte count}

The EFS in correlation with initial leukocyte count is depicted in Table 8; due to the small number of patients, we could not appreciate the statistical significance of the obvious differences between the 3 groups of patients.

TABLE 8. EFS related to initial leukocyte count

\begin{tabular}{|c|c|c|}
\hline Initial leukocyte count & $\begin{array}{c}\text { Number of } \\
\text { patients }\end{array}$ & $\begin{array}{c}\text { EFS at } \mathbf{4 0} \text { months } \\
\text { (\%) }\end{array}$ \\
\hline$<20,000 / \mu \mathrm{L}$ & 16 & 80 \\
\hline $20,000-100,000 / \mu \mathrm{L}$ & 9 & 62.5 \\
\hline$>100,000 / \mu \mathrm{L}$ & 7 & 55.6 \\
\hline
\end{tabular}

\section{Immunophenotype of the blast cells}

Distribution of phenotype in relation to the patient's age is presented in Figure 3.

Table 9 shows the EFS in our cohort of patients depending on the immunophenotype of the blast cells.

TABLE 9. EFS correlated with the immunophenotype of the blast cells

\begin{tabular}{|c|c|c|}
\hline Immunophenotype & $\begin{array}{c}\text { Number of } \\
\text { patients }\end{array}$ & $\begin{array}{c}\text { EFS at 40 months } \\
\text { (\%) }\end{array}$ \\
\hline B-common & 14 & 81 \\
\hline Pre-B & 3 & 71.4 \\
\hline T & 2 & 50 \\
\hline Pro-B & 3 & 33.3 \\
\hline
\end{tabular}




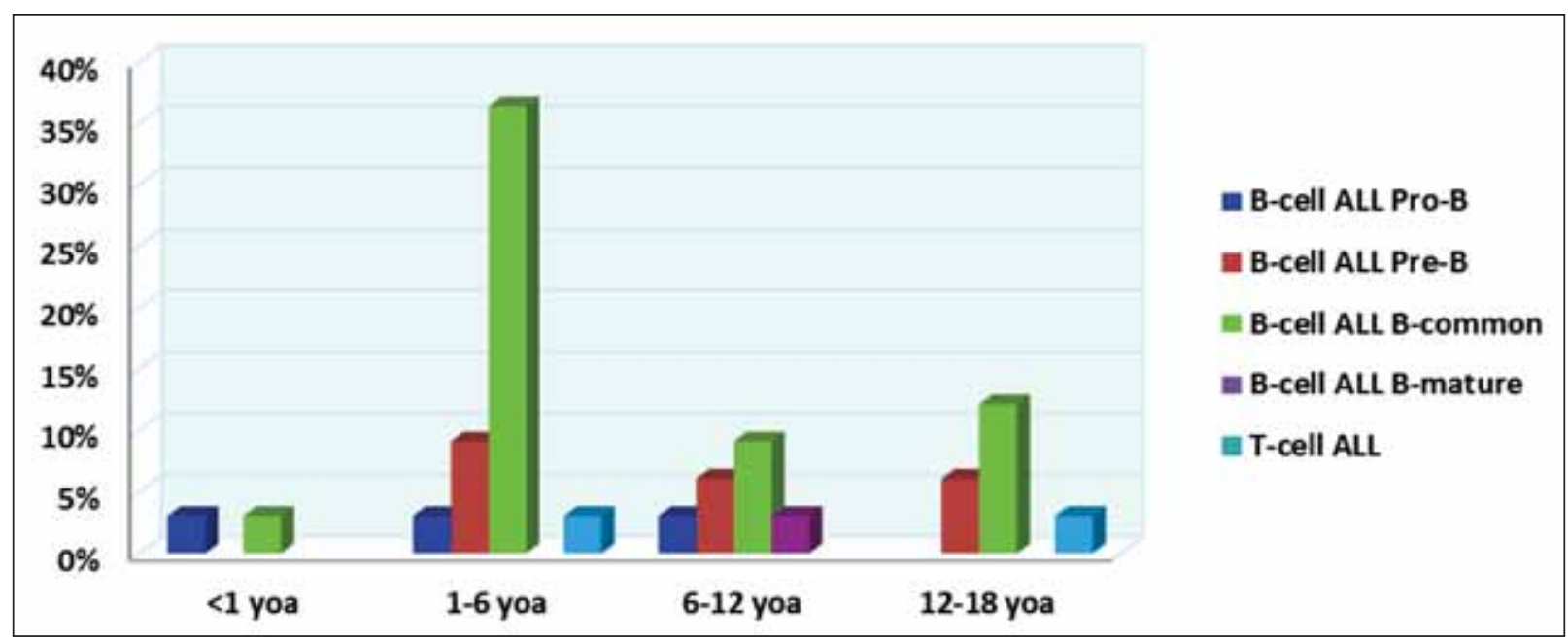

FIGURE 3. Correlation between blasts immunophenotype and the patient's age

\subsection{Allocation of patients to the risk groups in accordance with ALL IC-BFM 2002 and Interfant-06 Protocols of chemotherapy}

We present in Figure 4, the allocation of our patients to the risk groups according to the two Protocols used in this study.

The criteria for allocation are depicted in Table 3 and Table 4.

We have to mention that in the IR group we noted 5 patients with initial age 1-6 years, but initial leukocyte count was $>20,000 / \mu \mathrm{L} ; 7$ patients have initial age $>6$ yoa, but with on initial leukocyte count $<20,000 / \mu \mathrm{L}$; the other 2 patients from this group fulfilled both criteria: initial age $>6$ yoa, and initial leukocyte count $>20,000 / \mu \mathrm{L}$.

Discussing about the allocation of HR patients, we have to emphasize the next remarks:

2 patients had $\mathrm{t}(4 ; 11)(\mathrm{MLL}-\mathrm{AF} 4)$ translocation, one of them being PGR (prednisone good responder) and obtaining CR (complete remission) in bone marrow D33, and the other one PPR (prednisone poor responder) - did not obtain CR D33.
4 patients had $t(9 ; 22)$ (BCR-ABL) translocation, wo being PGR and obtaining CR D33; the others were poor prednisone responders, only one with CR D33 (for the second PPR it was an induction failure).

4 patients were PPR, with/without non-specific for HR cytogenetic abnormalities, two obtaining CR D33; for the rest, we noted induction failure.

\subsection{OS and EFS correlated with the risk group are presented in Table 10.}

\subsection{OS and EFS in correlation with risk group and associated cytogenetic/molecular abnormalities - Figure 5}

TABLE 10. OS and EFS correlated with the risk group of patients*

\begin{tabular}{|c|c|c|c|c|c|}
\hline \multicolumn{6}{|c|}{ Outcome at the end of study } \\
\hline \multicolumn{3}{|c|}{ OS (\%) } & \multicolumn{3}{|c|}{ EFS (\%) } \\
\hline SR & IR & HR & SR & IR & HR \\
\hline 100 & 88.89 & 60 & 88.89 & 85.7 & 60 \\
\hline
\end{tabular}

*OS by 40 months in the whole cohort of patients: $90.9 \%$; EFS by 40 months in the whole cohort of patients: $72.7 \%$.

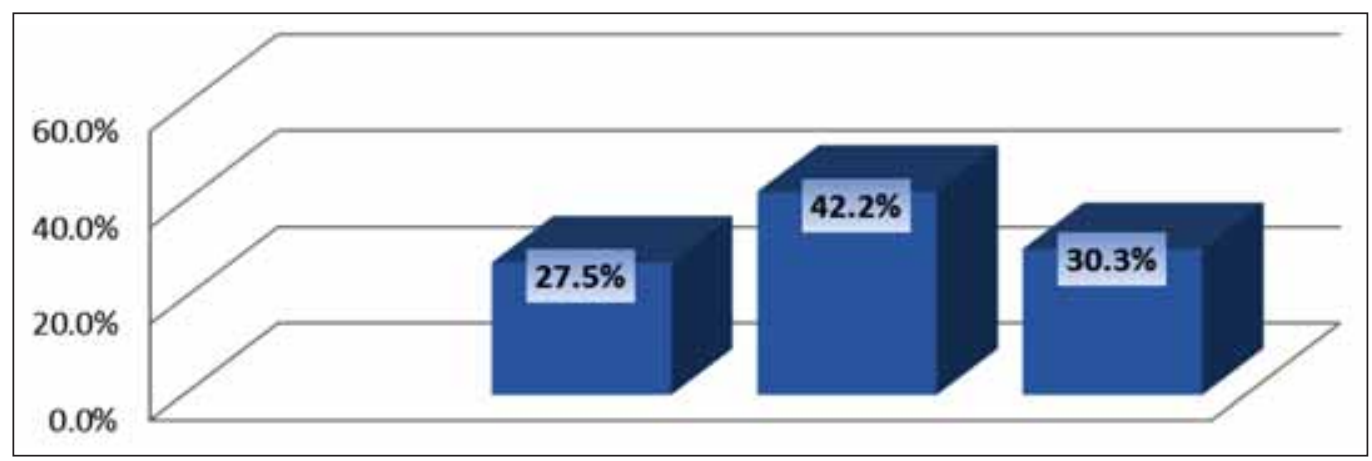

FIGURE 4. Allocation of patients to risk group 


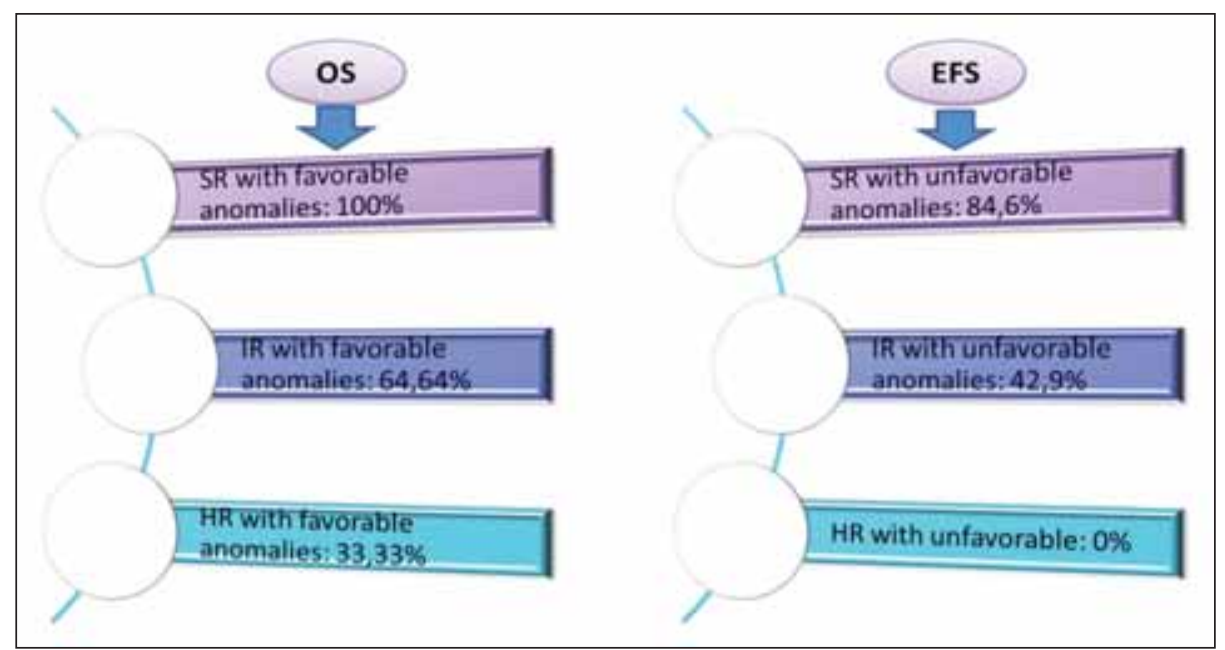

FIGURE 5. OS and EFS in correlation with risk group and associated cytogenetic/molecular abnormalities

Abbreviations: SR: standard risk; IR: intermediate risk; HR: High risk; OS: overall survival; EFS: event-free survival.

We considered favorable abnormalities like hyperdiploidy, $t(12 ; 21)$ (TEL-AML1) and $t(1 ; 14)$ (SIL-TAL1), the last one being specific only for T-cell ALL.

Unfavorable abnormalities in our cohort of patients were: hypodiploidy, t(9;22)(BCR-ABL), $\mathrm{t}(4 ; 11)(\mathrm{MLL}-\mathrm{AF} 4)$ and other MLL abnormalities.

We have also to mention that OS and EFS are estimated at 40 months of observation and that - due to small number of patients - we could not calculate the statistical significance, although the differences are obvious.

\subsection{Outcome in correlation with response to therapy}

The main check points related to response to therapy in the Protocols we used in this study were:

\subsubsection{Initial response to cortisone (D8 of induction)}

In our cohort of patients, they were 7 prednisone poor responders (PPR); it means a number of blast cells in peripheral blood $>1,000 / \mu \mathrm{L}$. The evolution of these patients is presented in Figure 6.

From a practical point of view, poor initial response to cortisone compels the inclusion of patients in the HR group and intensification of therapy.

\subsubsection{BM status $D 33$}

BM status M0, M1 in the D33 of induction means an appropriate response to induction therapy; BM status $\mathrm{M} 2, \mathrm{M} 3$ is the sign of induc- tion failure. In this study, 26 patients (78. 8\%) were in complete remission by the end of induction. Induction failure is a Protocol indication to move the patient in the high risk group and to further intensify the therapy.

The evolution of the patients in continuation of Protocol in correlation with their allocation to one of the risk groups is presented in Figure 7.

\subsubsection{Minimal residual disease}

MRD (by immunophenotyping or by molecular biology techniques - FISH and RT-PCR) was assessed in $10 / 33$ patients (33.33\%), only these having anomalies which could be demonstrated by the techniques we used. MRD was searched in two time points: TP1 (BM, D33 of induction) and TP2 (BM, D78 of therapy, before the initiation of Protocol M or the first HR block of chemotherapy).

In the standard risk group, MRD was assessed in 3 patients with $t(12 ; 21)$ (TEL-AML1) translocation. We could demonstrate the clearance of blasts in BM at the end of induction in all 3 patients (in one after the intensification of therapy). All these 3 patients remained in $C R$ at the end of study.

In the high risk group, all the patients had cytogenetic or molecular abnormalities who could enable us to discover the MRD. MRD detected by FISH in 2 cases with $\mathrm{t}(4 ; 11)$ (MLL-AF4) translocations was persistent by the both check points; at the end of study the evolution was unfavorable in these patients.

MRD was also tested in $3 \mathrm{HR}$ patients with $t(9 ; 22)(B C R-A B L)$ and $t(4 ; 11)(M L L-A F 4)$ translocation. Only in one patient with BCR-ABL product 


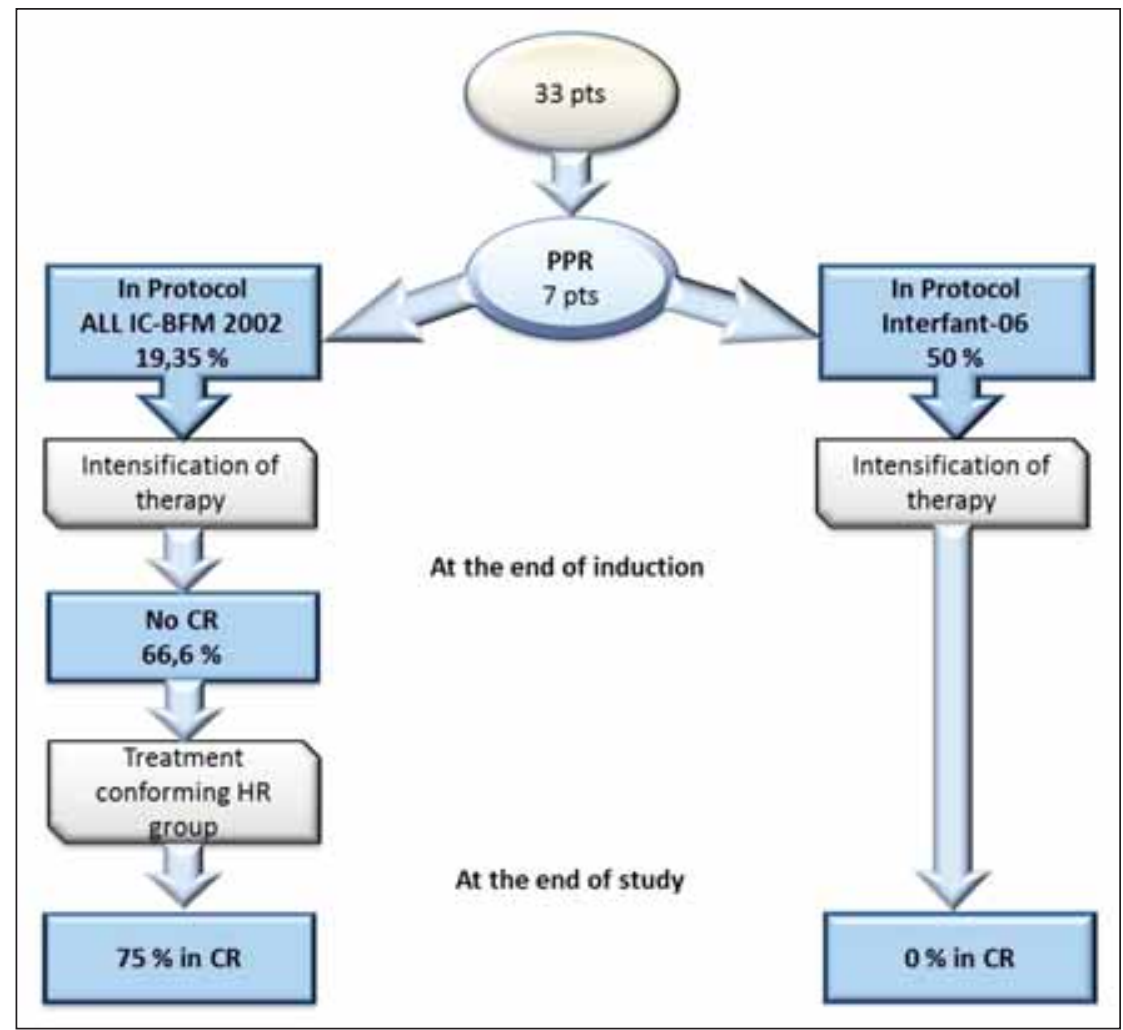

FIGURE 6. Evolution of prednisone poor responders

Abbreviations: PPR: prednisone poor responders; CR: complete remission.

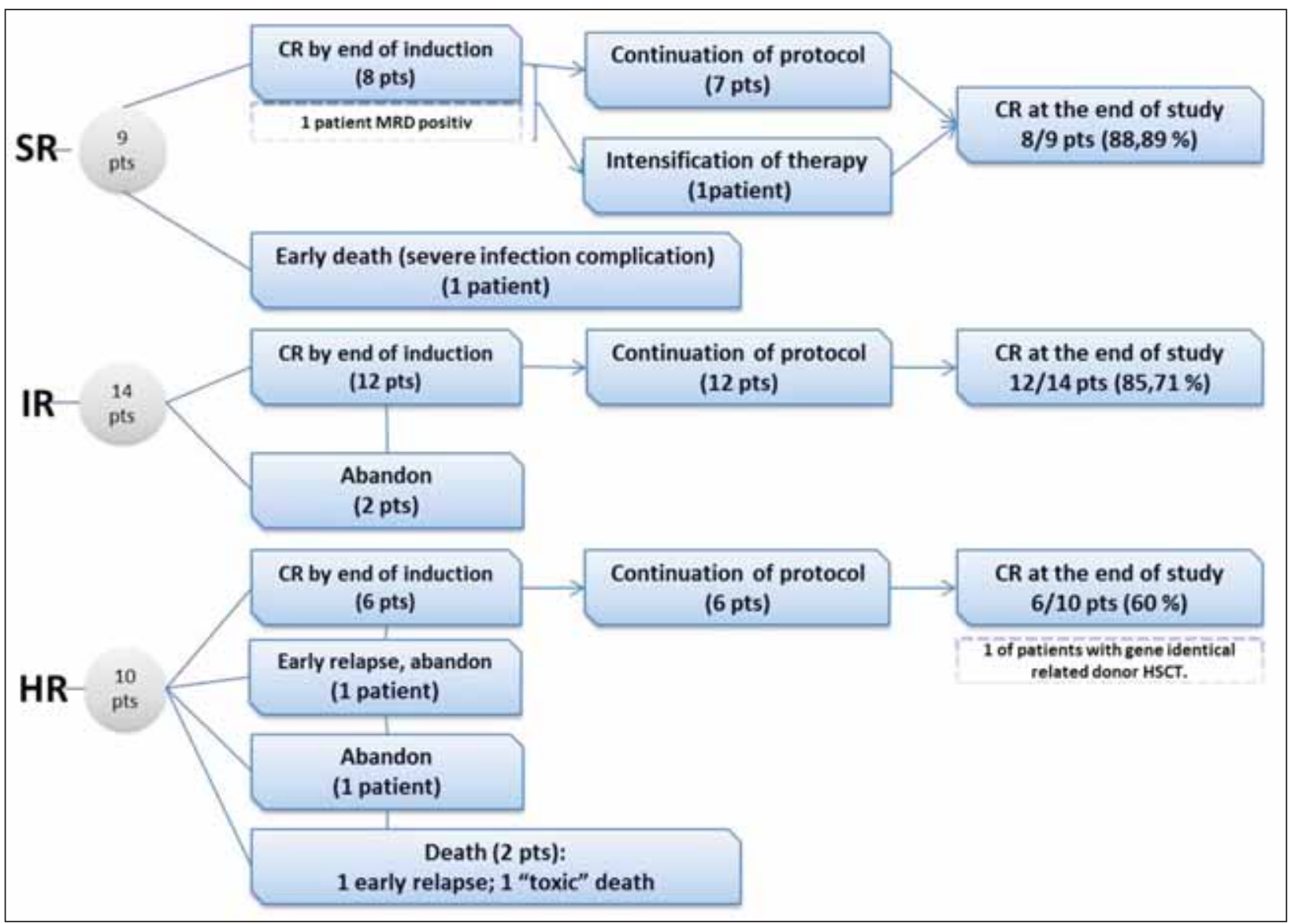

FIGURE 7. Evolution of ALL patients in correlation to risk groups and the BM status D33 of Protocol

Abbreviations: pts: patients; SR: standard risk; IR: intermediate risk; HR: high risk; CR: complete remission; MRD: minimal residual disease; HSCT: hematopoietic stem cell transplantation. 
of fusion and treated by chemotherapy and tyrosine kinase inhibitor (TKI) - Imatinib in association, MRD was negative in both check points and final evolution was favorable. In 2 cases with $t(4 ; 11)$ translocation, MRD was positive and final outcome unfavorable.

\section{Primary and secondary complications related to patient's risk group}

We considered primary complications the events due to evolution of the disease, and secondary those due to infections, toxic, metabolic causes, etc. Table 11 summarizes the main complications in our cohort of patients.

\section{DISCUSSION}

ALL in children is considered now a highly curable cancer; OS is $90 \%$ in the most privileged countries. $(2,20)$ This was achieved by escalating the intensity of treatments - chemotherapy and HSCT (hematopoietic stem cell transplantation) - but it is related to a much more toxicity of therapy.

The major events who are the backbone on actual therapy are risk adapted treatment (based on biological features of the disease), CNS prophylaxy and the improvement of supportive care. The modern ALL therapy is based on risk stratification, up front, at diagnose and later on and by response to therapy in the different phases of Protocols.

One of the main aim of our study, was to assess the relationship between principal biological characteristics of the disease and the therapeutical outcome. In this study, we were able to demonstrate the value for predicting the response to therapy of such biological factors as:

Age at diagnosis - the best prognosis was in the 1-6 yoa group, while the ALL children aged less than $1 \mathrm{y}$ at diagnose had the worst prognosis;

Initial leukocyte count $(<20,000 / \mu \mathrm{L}$ : $80 \%$ EFS at $40 \mathrm{Mo}$, between $20,000-100,000 / \mu \mathrm{L}$ : $62.5 \%$ and $>100,000 / \mu \mathrm{L}$ only $55.6 \% \mathrm{EFS}$ at $40 \mathrm{Mo}$ );

Blast's immunophenotype, with B-common ALL having the best outcome ( $81 \%$ EFS by 40 $\mathrm{Mo}$ ), and pro-B the worst one (only $33.3 \% \mathrm{EFS}$ by $40 \mathrm{Mo}$ );

Associated cytogenetic and molecular anomalies - The impact of these biological variables is visible especially for HR group patients. In our cohort, EFS was $33.33 \%$ in HR patients with favorable abnormalities (hyperdiploidy, $\mathrm{t}(12 ; 21)$ (TEL-AML1), and t(1;14)(SIL-TAL1)), the last one
TABLE 11. Primary and secondary complications according to risk groups

\begin{tabular}{|l|c|c|c|}
\multicolumn{1}{|c|}{ Risk group } & $\begin{array}{c}\text { SR } \\
\text { (Nr. pts) }\end{array}$ & $\begin{array}{c}\text { IR } \\
\text { (Nr. pts) }\end{array}$ & $\begin{array}{c}\text { HR } \\
\text { (Nr. pts) }\end{array}$ \\
\hline Testicular involvement & - & 1 & - \\
\hline Bone pain/skeletal & - & - & 1 \\
\hline Infections URTI & 4 & 5 & 8 \\
\hline Infections (bronchopneumonia) & 1 & 1 & - \\
\hline Facial, skin, soft tissue infections & - & 2 & - \\
\hline Herpes & 2 & 1 & 2 \\
\hline Tumor lysis syndrome & - & - & 2 \\
\hline \multicolumn{1}{|c|}{ Secondary complication } \\
(mostly due to therapy) & & & \\
\hline BM aplasia (post-CxT) & & & \\
\hline Cushing syndrome & 9 & 14 & 10 \\
\hline Mucositis & 9 & 14 & 10 \\
\hline Gastritis & 9 & 14 & 10 \\
\hline Myopathy & 5 & 8 & 4 \\
\hline Osteoporosis & 6 & 8 & 5 \\
\hline ADH inadequate secretion & - & 1 & 2 \\
\hline DVT / stroke & - & 2 & 2 \\
\hline Hypofibrinogenemia(L-ASP) & - & 2 & 2 \\
\hline Secondary hypertension & 1 & 3 & 1 \\
\hline Hypercholesterolemia & - & 1 & 1 \\
\hline Hepatotoxicity & 1 & 3 & 3 \\
\hline Severe hemorrhage & 5 & 8 & 2 \\
\hline Erythema polymorphe & - & 2 & 3 \\
\hline URTI & - & 1 & - \\
\hline Bronchitis & 3 & 5 & 4 \\
\hline Pneumonia \pm ARDS & 3 & 4 & 1 \\
\hline UTI & 3 & 3 & 5 \\
\hline HSV infection & 2 & 1 & 2 \\
\hline Bacterial sepsis & 1 & 2 & 2 \\
\hline Fungal infection & 2 & 1 & 6 \\
\hline CVC infection & - & 2 & 1 \\
\hline MAS & - & 1 & 1 \\
\hline ALF & - & - & 1 \\
\hline ARF & 1 & - & 1 \\
\hline N VII paralysis & - & 1 & - \\
\hline Abbreviations: URTI: & - & - & 2 \\
\hline
\end{tabular}

Abbreviations: URTI: upper respiratory tract infection; BM: bone marrow; CXT: chemotherapy; HSV: herpes simplex virus; ADH: antidiuretic hormone; DVT: deep vein thrombosis; L-ASP: L-asparaginase; ARDS: adult respiratory distress syndrome; UTI: urinary tract infection; CVC: central venous catheters; MAS: macrophage activation syndrome; ALF: acute liver failure; ARF: acute renal failure; N VII: facial nerve.

being specific for T-cell ALL, whereas in association with unfavorable abnormalities $(t(9 ; 22)$ (BCR-ABL), $t(4 ; 11)$ (MLL-AF4) and hypodiploidy) HR patients had $E F S=0$.

Regarding to estimation of response to therapy as another major factor with impact on pediatric ALL prognosis, we would like only to emphasize the value of such factors as:

Initial response to cortisone by D8 of induction (from 7 PPRs after intensification of therapy treating them with HR ALL IC-BFM 2002 Protocol, only 3 patients were in $C R$ at the end of study);

BM status by D33 of Induction (BM-M0, M1 by D33 indicates an appropriate response to therapy andgood prognosis, while BM-M2, M3 
means induction failure and poor prognosis). Induction failure is an indication of most recent Protocols (ALL IC-BFM 2002, Interfant 06) to assign the patient to HR group and to further intensify the therapy.

MRD was assessed by multicolor flow cytometry, immunophenotyping or by molecular techniques (FISH and RT-PCR) on 2 time points (D33 and D78 of therapy). Demonstration of blast's clearance by these two time points is a factor of good prognosis, whereas MRD positivity predicts an unfavorable outcome.

We have to emphasize the fact that the results of our study are similar to the conclusions of other contemporary studies. (21-26) Also, we should to mention that the major limitations of personal study are the relative small number of patients ( $33 \mathrm{pts}$ ), and the limited period of observation (only 40 months); these would not enable us to assess the statistical significance, even the most differences are obvious.

From a more general print of view, the validity of our study was also demonstrated by different outcomes related to allocation of patients to risk groups, which are mostly based on variables above discussed: EFS by $40 \mathrm{Mo}$ being $88.89 \%, 85.7 \%$ and $60 \%$ for SR, IR and HR groups respectively.

An argument in favor of increased toxicity of contemporary Protocols of chemotherapy is the list of secondary complications in our cohort of patients (Table 11).

The major challenges for the development of newer, and better Protocols for treatment in pediatric ALL remain: the adverse prognosis of infant ALL, the role in leukemogenesis and the peculiarities of constitutional predispositions, some biological anomalies (like hypo-diploid ALL, ALL with iAMP21 (intrachromosomal amplification of chromosome 21), BCR-ABL like pe- diatric ALL, early T-cell precursor ALL), as well as induction failure, refinement of MRD diagnose, and the new targeted therapies. (27)

For our country the main problems remain: the better endowment of laboratories and of clinical wards, as well as a better co-operation between national pediatric centers and a more active participation at international Protocol studies, in order to mitigate the differences between outcomes in comparison to Center from higher income countries.

\section{CONCLUSION}

The use of modern Protocols of chemotherapy in pediatric ALL lead to the assumption that this atrocious disease could be highly curable (more than $90 \%$ EFS was reported by some Centers).

Recent worldwide studies, as well as our study on a small cohort of 33 consecutive patients emphasize the value of such biological factors for the better use the current treatment modalities, as initial age of patients, leukocyte count at diagnosis, blast's immunophenotype, cytogenetics and molecular characteristics. Also, they demonstrate the impact of monitoring the evolution of patients on therapy - especially by MRD - for adapting the Protocols to patient's peculiarities.

We should to emphasize that remaina lot of major challenges for development of newer, more active, Protocols in pediatric ALL: the poor prognosis of infant as well as the adolescent ALL, some biological specificities of the disease like hypodiploid ALL, ALL with iAMP21, BCR-ABL like pediatric ALL, early T-cell precursor ALL, etc.

To solve all these challenges it is necessary the refinement of preclinical studies, as well as the amplification of cooperation between the Pediatric Hematology Oncology Centers.

\section{$\overline{\text { BIBLIOGRAFIE }}$}

1. Chaleff S. - Advances in prognostication and treatment of pediatric acute leukemia, Diagnostic. Cambridge: Cambridge University Press.

2. National Cancer Institute - Childhood Acute Lymphoblastic Leukemia Treatment (Online). Available: http://www.cancer.gov/ types/leukemia/hp/child-all-treatmentpdq\#section/all. (Accessed: 26-Sep-2015).

3. American Cancer Society - Childhood Leukemia Detailed Guide (Online).
Available: http://www.cancer.org/cancer/ leukemiainchildren/detailedguide/ leukemia-in-children-detailed-guide-toc. (Accessed: 03-Oct-2015).

4. Childhood Acute Lymphoblastic Leukemia: Diagnosis, Management, and Complications. (Online). Available: http:// reference.medscape.com/features/ slideshow/acute-lymphoblastic-leukemia\#3. (Accessed: 03-Oct-2015).

5. Pui C.-H., Howard S.C. - Current management and challenges of malignant disease in the CNS in paediatric leukaemia, Lancet. Oncol, vol. 9, no. 3, pp. 257-68, Mar. 2008.

6. Pui C.H., Campana D., Pei D., et al Treating childhood acute lymphoblastic leukemia without cranial irradiation, $N$ Engl J Med, vol. 360, no. 26, pp. 2730-2741, 2009.

7. Martin A., Morgan E., Hijiya N. - Relapsed or refractory pediatric acute lymphoblastic leukemia: current and emerging treatments, 
Paediatr. Drugs, vol. 14, no. 6, pp. 377-87, Dec. 2012.

8. Satwani P., Sather H., Ozkaynak F. et al - Allogeneic bone marrow transplantation in first remission for children with ultra-highrisk features of acute lymphoblastic leukemia: A children's oncology group study report, Biol. Blood Marrow Transplant, vol. 13, no. 2, pp. 218-27, Feb. 2007.

9. Dita C., Colita A., Asan M., Gheorghe A., Arion C. - Modern Diagnostic Approach of Acute Lymphoblastic Leukemia in Children and Adolescents - Experience of a Single Pediatric Hematology-Oncology Center, Mod. Med., vol. 22, no. 4, pp. 300-308, 2015.

10. Thörn I., Forestier E., Botling J., et al Minimal residual disease assessment in childhood acute lymphoblastic leukaemia: a Swedish multi-centre study comparing real-time polymerase chain reaction and multicolour flow cytometry, Br. J. Haematol., vol. 152, no. 6, pp. 743-53, Mar. 2011.

11. van Dongen J.J., Seriu T., Panzer-Grümayer E.R., et al - Prognostic value of minimal residual disease in acute lymphoblastic leukaemia in childhood, Lancet, vol. 352, no. 9142, pp. 1731-8, Nov. 1998.

12. Campana D. - Role of minimal residual disease monitoring in adult and pediatric acute lymphoblastic leukemia, Hematol. Oncol. Clin. North Am., vol. 23, no. 5, pp. 1083-98, vii, Oct. 2009.

13. Bhojwani D., Pui C.-H. - Relapsed childhood acute lymphoblastic leukaemia, Lancet. Oncol., vol. 14, no. 6, pp. e205-17, May 2013.
14. ALL-IC-BFM: A Randomized Trial of the I-BFM-SG for the Management of Childhood non-B Acute Lymphoblastic Leukemia, 2002.

15. Interfant 06: International collaborative treatment protocol for infants under one year with acute lymphoblastic or biphenotypic leukaemia, 2006.

16. World Medical Association - Declaration of Helsinki: ethical principles for medical research involving human subjects, JAMA, vol. 310, no. 20, pp. 2191-4, Nov. 2013.

17. Bene M.C., Castoldi G., Knapp W., et al - Proposals for the immunological classification of acute leukemias. European Group for the Immunological Characterization of Leukemias (EGIL), Leukemia, vol. 9, no. 10, pp. 1783-6, Oct. 1995.

18. Hastie T., Tibshirani R. - Generalized additive models for medical research, Stat. Methods Med. Res., vol. 4, no. 3, pp. 187-196, 1995.

19. Klein J.P., Moeschberger M.L. - Survival Analysis: Techniques for Censored and Truncated Data, 2nd ed. Springer, 2003.

20. Hunger S.P., Lu X., Devidas M., et al Improved survival for children and adolescents with acute lymphoblastic leukemia between 1990 and 2005: a report from the children's oncology group, J. Clin. Oncol, vol. 30, no. 14, pp. 1663-9, May 2012.

21. Pui C.-H., Pei D., Campana D., et al Improved prognosis for older adolescents with acute lymphoblastic leukemia, J. Clin. Oncol, vol. 29, no. 4, pp. 386-91, Feb. 2011.
22. Arico M., Schrappe M., Hunger S.P., et al Clinical Outcome of Children With Newly Diagnosed Philadelphia ChromosomePositive Acute Lymphoblastic Leukemia Treated Between 1995 and 2005, J. Clin. Oncol, vol. 28, no. 31, pp. 4755-4761, Sep. 2010.

23. Nachman J.B., Heerema N.A., Sather H., et al - Outcome of treatment in children with hypodiploid acute lymphoblastic leukemia, Blood, vol. 110, no. 4, pp. 1112-5, Aug. 2007.

24. Roberts K.G., Pei D., Campana D. et al Outcomes of children with BCR-ABL1-like acute lymphoblastic leukemia treated with risk-directed therapy based on the levels of minimal residual disease, J. Clin. Oncol, vol. 32, no. 27, pp. 3012-20, Sep. 2014.

25. Vora A., Goulden N., Mitchell C. et al Augmented post-remission therapy for a minimal residual disease-defined high-risk subgroup of children and young people with clinical standard-risk and intermediate-risk acute lymphoblastic leukaemia (UKALL 2003): a randomised controlled trial, Lancet. Oncol, vol. 15, no. 8, pp. 809-18, Jul. 2014.

26. Schrappe M.O., Hunger S.P., Pui C.H., et al - Outcomes after induction failure in childhood acute lymphoblastic leukemia, N. Engl. J. Med., vol. 366, no. 15, pp. 1371-81, Apr. 2012.

27. Baruchel A. - Remaining challenges in pediatric and adolescent acute lymphoblastic leukemia, Hematol. Educ. Educ. Progr. Annu. Congr. Eur. Hematol. Assoc., vol. 9, no. 1, pp. 17-23, 2015. 